\title{
Inhibitory effects of ultrasound irradiation on Staphylococcus epidermidis biofilm
}

\author{
Harumi Koibuchi ${ }^{1}$ (1) Yasutomo Fujii ${ }^{2} \cdot$ Yusuke Sato'o $^{3} \cdot$ Takashi Mochizuki $^{4} \cdot$ Toshiyuki Yamada $^{1} \cdot$ Longzhu Cui $^{3}$. \\ Nobuyuki Taniguchi ${ }^{1}$
}

Received: 23 November 2020 / Accepted: 2 July 2021 / Published online: 19 August 2021

(c) The Author(s) 2021, corrected publication 2021

\begin{abstract}
Purpose We aimed to investigate whether low-intensity continuous and pulsed wave ultrasound (US) irradiation can inhibit the formation of Staphylococcus epidermidis biofilms, for potential application in the treatment of catheter-related bloodstream infections (CRBSI).

Methods S. epidermidis biofilms that formed on the bottom surfaces of 6-well plates were irradiated on the bottom surface using the sound cell incubator system for different intervals of time.

Results US irradiation with continuous waves for $24 \mathrm{~h}$ notably inhibited biofilm formation $(p<0.01)$, but the same US irradiation for $12 \mathrm{~h}$ had no remarkable effect. Further, double US irradiation with pulsed waves for 20 min inhibited biofilm formation by $33.6 \%$, nearly two-fold more than single US irradiation, which reduced it by $17.9 \%$.

Conclusion US irradiation of a lower intensity $\left(I_{\text {SATA }}=6-29 \mathrm{~mW} / \mathrm{cm}^{2}\right)$ than used in a previous study and lower than recommended by the Food and Drug Administration shows potential for preventing CRBSI caused by bacterial biofilms.
\end{abstract}

Keywords Biofilm · Staphylococcus epidermidis · Ultrasound · Catheter-related bloodstream infection · Acoustic parameter

\section{Introduction}

The use of central venous catheterization has become important in the management of critical patients. Insertion of central venous catheters makes vascular access secure [1]. Catheter-related bloodstream infection (CRBSI) is a serious infectious disease in hospitalized patients because it increases the length of stay, cost of care, and risk of hospital death [2]. The cost of CRBSI ranges between $\$ 33,000$ and $\$ 75,000$, depending on the type of intensive care unit [3]. The most common causative agents of CRBSI are coagulasenegative staphylococci such as Staphylococcus epidermidis

Harumi Koibuchi

koichan@jichi.ac.jp

1 Department of Clinical Laboratory Medicine, Jichi Medical University, 3311-1 Yakushiji Shimotsuke-Shi, Tochigi 329-0498, Japan

2 Department of Human Health Science, Kyoto University Graduate School of Medicine, Kyoto, Japan

3 Division of Bacteriology, Department of Infection and Immunity, Jichi Medical University, Tochigi, Japan

4 Medical Ultrasound Laboratory Co., Ltd., Tokyo, Japan
(S. epidermidis). This bacterium ubiquitously colonizes the skin and invades the bloodstream through skin-inserted medical devices, such as intravascular catheters and joint prostheses [4]. Biofilm formation is essential for the pathogenicity of $S$. epidermidis [5]. The biofilm matrix prevents the access of antibiotics to bacterial cells. Alternatively, the slow growth of bacteria in the mature biofilm protects the cells from antibiotics [6]. Therefore, biofilm-forming bacteria are exceptionally resistant to antibiotics, and biofilmassociated infection is one of the most serious problems in hospitalized patients.

Ultrasound (US) energy has a chemical and biological effect on cells [7]. Considerable attention should be paid to US intensity when US irradiation is used for therapeutic purposes in clinical settings because US energy has biological effects on normal cells. The Food and Drug Administration (FDA) has recommended guidelines for the output of diagnostic ultrasound medical devices. This guideline recommends an upper limit level of US intensity of $720 \mathrm{~mW} / \mathrm{cm}^{2}$ $\left(I_{\mathrm{SPTA}}\right)$ on peripheral vessels [8].

The effectiveness of US irradiation in biofilm eradication has been reported previously; however, the US intensity used in these studies is too high according to FDA guidelines for 
clinical therapy applications $[9,10]$. In our previous study, US irradiation decreased the amount of $S$. epidermidis biofilm produced on the bottom surface of wells in a 6-well plate [11]. The biofilm was irradiated with $1-\mathrm{MHz}$ continuous-wave US in this study. US intensity was $I_{\mathrm{SPTA}}=1.66$ $\mathrm{mW} / \mathrm{cm}^{2}$, and the irradiation time was $24 \mathrm{~h}$. This intensity was lower and the time was longer than those in other similar studies $[9,10]$.

Furthermore, previous studies focused on using US irradiation for the eradication of formed biofilms. However, if a biofilm is destroyed by US irradiation, bacteria present in and around the biofilm may disperse into the bloodstream and cause bacteremia in the patient. Therefore, for treating CRBSI, it is important to prevent bacteria from forming a biofilm rather than destroying a completely formed biofilm.

In this study, we used sound cell incubator (SCI) as a US irradiation system. The purpose of the study was to evaluate the inhibitory effect of US irradiation with a lower intensity level than that used in previous studies on biofilm formation.

\section{Materials and methods}

\section{Preparation of bacterial solution}

S. epidermidis (ATCC 35984 RP 62A) was purchased from American Type Culture Collection (ATCC, Virginia, USA) and stocked in Microbank (Iwaki, Tokyo, Japan). Bacterial cells were inoculated on Tryptic Soy Agar plates (Becton-Dickinson and Company, Sparks, USA) at $37^{\circ} \mathrm{C}$ in $5 \%$ $\mathrm{CO}_{2}$ for $24 \mathrm{~h}$. We inoculated five or six colonies of $S$. epidermidis in $5 \mathrm{~mL}$ of Brain Heart Infusion (BHI, Becton-Dickinson and Company) in 15-mL Corning tubes (Corning, Glendale, Arizona, USA). The tubes were incubated at $37{ }^{\circ} \mathrm{C}$ in $5 \% \mathrm{CO}_{2}$ for $18 \mathrm{~h}$.

\section{Biofilm grown in 6-well plates}

Twenty $\mu \mathrm{L}$ of bacterial culture was added to $2 \mathrm{~mL}$ of BHI containing 1\% D-glucose (Wako, Osaka, Japan) in 6-well plates (Thermo Fisher Scientific, NY, USA). After incubation, media were discarded and the wells were washed four times using physiological saline. White patches left on the bottom surfaces of the wells after washing were considered "biofilm."

\section{Quantification of biofilms}

The amount of biofilm was quantified by crystal violet staining. The biofilm was stained with $2 \mathrm{~mL}$ of $1 \%(\mathrm{w} / \mathrm{v}$ ) crystal violet (Merck KGaA, Darmstadt, Germany) for 30 min. After incubation, the dye was discarded and the wells were gently washed twice with distilled water. To extract the stain, a mixture of $1 \%(\mathrm{v} / \mathrm{v})$ hydrochloric acid and $70 \%(\mathrm{v} / \mathrm{v})$ ethanol was added to the wells and the extracted solutions were diluted 20 times. The amount of biofilm was determined by measuring the absorbance of the diluted solution at $595 \mathrm{~nm}$.

\section{US irradiation system}

For ultrasound irradiation of cells in this study, the SCI system (Medical Ultrasound Laboratory, Tokyo, Japan) was employed, in which essential irradiation parameters such as ultrasound frequency, pulse repetition time (PRT), pulse duty ratio, duration of irradiation, and driving voltage can be easily and precisely set up as requested. The system also has a unique feature where the irradiation of cells shown in Fig. 1a could be carried out inside the cell incubator under low-intensity ultrasound and long duration of irradiation. The SCI system is shown in Fig. 1b. Figure 1c is a schematic block diagram of the SCI system.

\section{Measurement of US irradiation intensity}

As it was expected that multiple echoes and standing waves would be generated in this US irradiation system for biofilm, US intensity was measured using the following method. Degassed water was put into a well of a 6-well plate. A hydrophone connected to an oscilloscope was set inside the well of the plate placed on the SCI irradiator. Subsequently, the hydrophone was moved along the well diameter in the $X$ and $Y$ directions to obtain the distribution of sound pressure in the well. Using the distribution, the spatial-averagetemporal-average intensity $\left(I_{\text {SATA }}\right)$ was estimated (Fig. 2a, b).

\section{Examination 1}

\section{Inhibitory effect of long-time US irradiation on biofilm formation}

Twenty $\mu \mathrm{L}$ of bacterial culture was added to $2 \mathrm{~mL}$ of BHI containing $1 \%$ glucose. The culture was then distributed in wells of two 6-well plates. One plate was for US irradiation and the other was for non-US irradiation as a control. US irradiation was performed using the SCI irradiator in an incubator $\left(37{ }^{\circ} \mathrm{C}\right.$ and $\left.5 \% \mathrm{CO}_{2}\right)$. The plate for non-US irradiation was put in the incubator without US irradiation.

US irradiation was of the continuous wave type, and its frequency was $1 \mathrm{MHz}$. US irradiation time was set at 24 and $12 \mathrm{~h}$. After US irradiation, the amount of biofilm produced on the bottom surfaces of the wells was assessed by crystal violet staining as described earlier.

This examination was repeated twice on other days, and 12 samples were ultimately obtained. 
Fig. 1 a US irradiation can be carried out in the cell incubator by SCI. b This system consists of driving unit SCI-D100, a personal computer with software to control it, plus and minus power supply units, and irradiator SCI-m1-6 (put on the $\mathrm{PC}$ in the photogram) for irradiation of a 6-well plate. $\mathrm{c}$ A schematic block diagram of the SCI system-which has four independent blocks of driving electric circuits, each of which consists of a logic generator and a pulse transmitter-is shown. Accordingly, one run of the experiment can be done under four different irradiation conditions at the same time
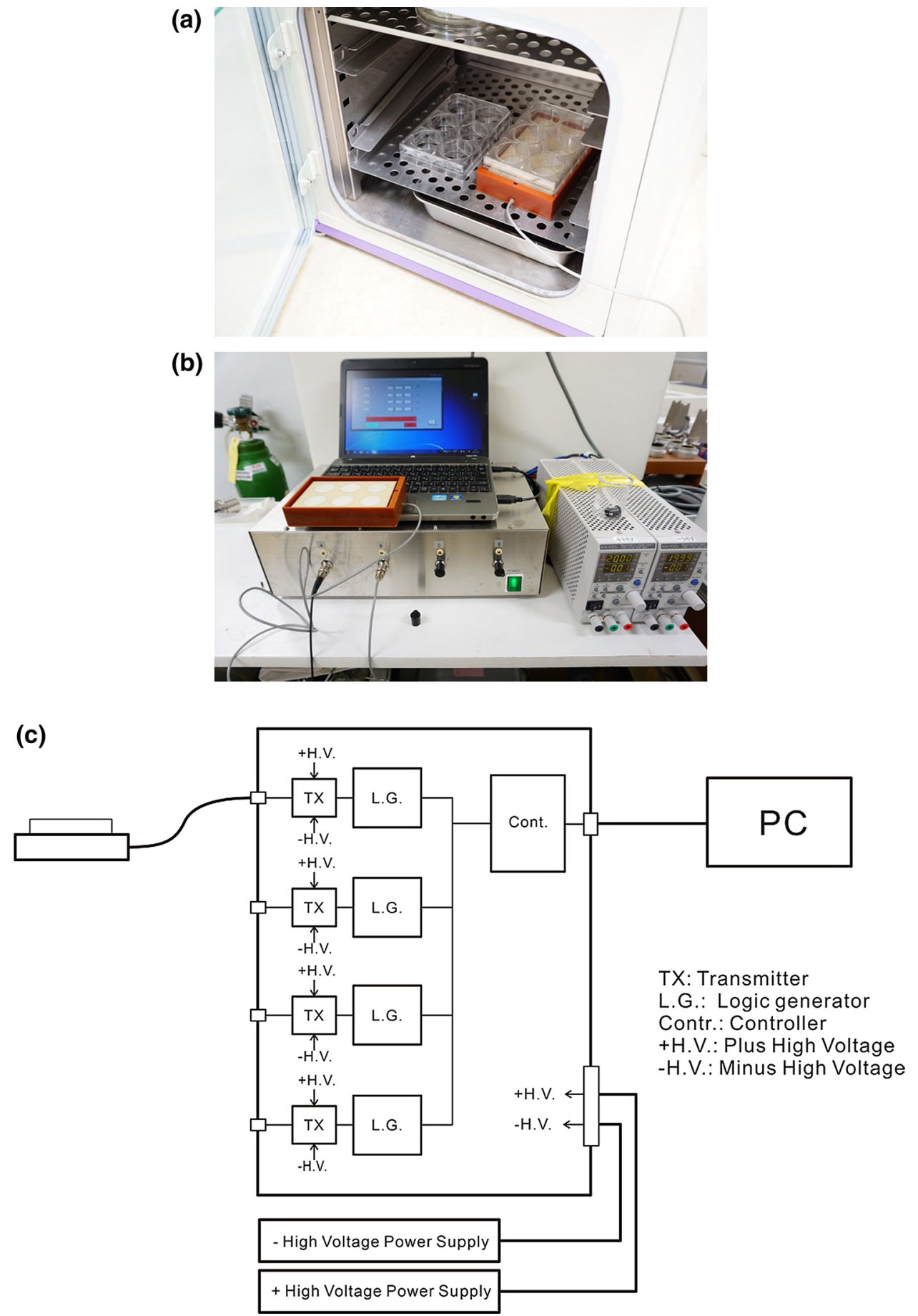

\section{Examination 2}

\section{Inhibitory effect of short-time US irradiation on biofilm formation}

In this examination, the parameters of US irradiation were set according to a previous report that described US irradiation on osteoblasts and showed that irradiation with lowintensity pulsed US enhanced bone fracture healing [12].
In this case, US irradiation parameters were as follows: US intensity, $30 \mathrm{~mW} / \mathrm{cm}^{2}$; irradiation time, $20 \mathrm{~min}$; US frequency, $1 \mathrm{MHz}$; and duty ratio, $20 \%$.

Twenty $\mu \mathrm{L}$ of bacterial culture was added to $2 \mathrm{~mL}$ of BHI containing $1 \%$ glucose. The culture was then distributed in wells of two 6-well plates. The plate for US irradiation was put on the SCI irradiator, and the SCI irradiator along with the plate was put in the incubator set at $37^{\circ} \mathrm{C}$ and $5 \% \mathrm{CO}_{2}$. The plate for non-US irradiation was put in 
Fig. 2 The schema for the method of measurement of ultrasound (US) intensity. The hydrophone was moved in the well filled with degassed water

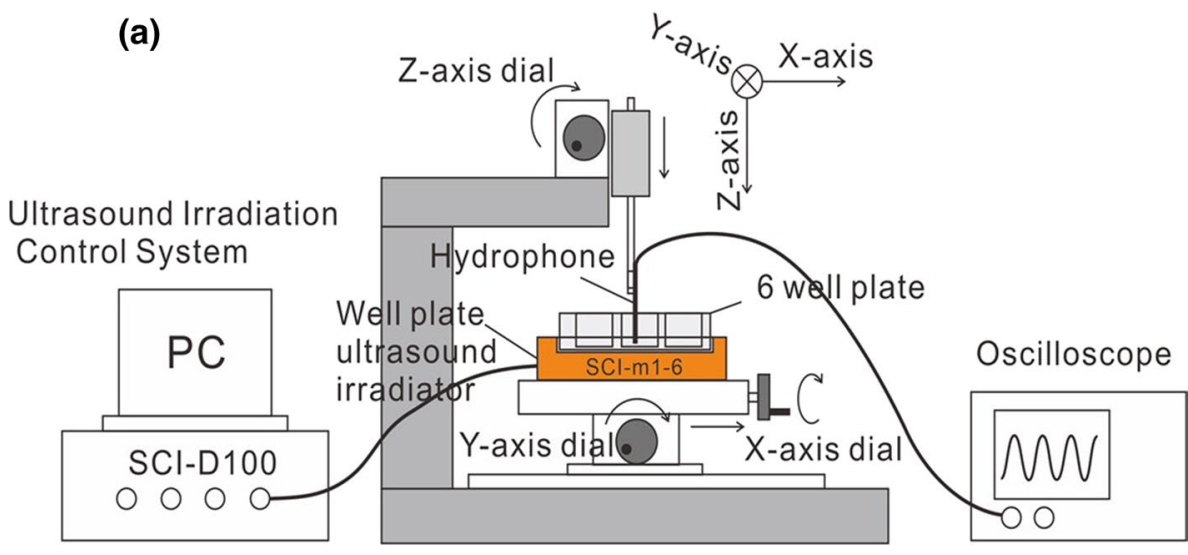

(b)

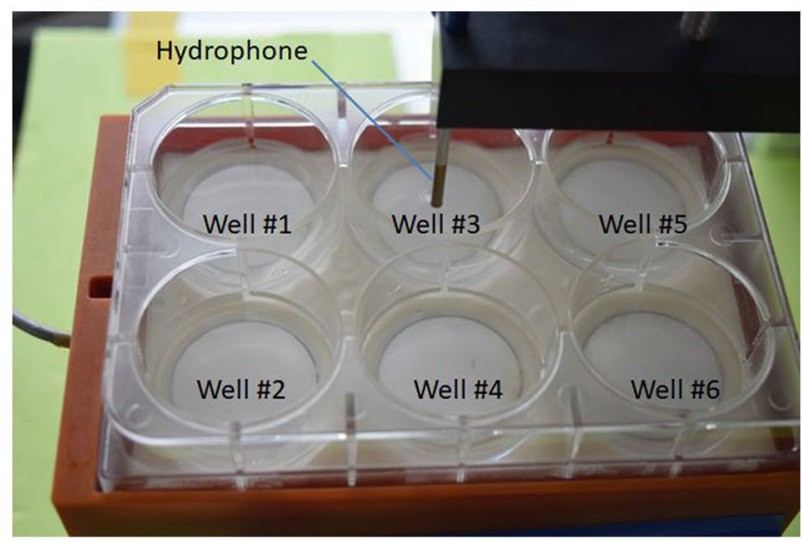

the same incubator at the same time. After one hour, the plate for US irradiation was irradiated with the US for $20 \mathrm{~min}$. US irradiation parameters were as follows: US frequency, $1 \mathrm{MHz}$; PRT, $10 \mathrm{~ms}$; and duty ratio, 20\%. US irradiation was performed once for the single-irradiation group and twice for the double-irradiation group, with a 160-min interval. After this, the plates were further incubated at $37^{\circ} \mathrm{C}$ for $6 \mathrm{~h}$ for biofilm formation. The amount of biofilm formed was assessed by crystal violet staining as described earlier (Fig. 3a, b). This examination was repeated twice on other days, and 12 samples were obtained.

\section{Statistical analysis}

All statistical analyses were performed using IBM SPSS Statistics for Windows, version 24 (IBM Corp., Armonk, NY, USA). The absorbance of samples at $595 \mathrm{~nm}$ is shown as mean $\pm \mathrm{SD}$. Statistical significance between the absorbance of the US irradiation group and the non-US irradiation group was determined using a two-tailed unpaired Student's $t$-test, and values with $p<0.01$ were considered significant.

\section{Results}

\section{US intensity in this study}

In "Examination 1", the US intensity was $I_{\text {SATA }}=29 \mathrm{~mW} /$ $\mathrm{cm}^{2}$, whereas, in "Examination 2", it was $I_{\text {SATA }}=6 \mathrm{~mW} / \mathrm{cm}^{2}$ (Fig. 4a-e).

\section{Examination 1}

\section{US irradiation for $\mathbf{2 4} \mathrm{h}$ notably reduced biofilm formation}

For the samples irradiated for $24 \mathrm{~h}$, the mean absorbance was $0.411 \pm 0.099$ in the US irradiation group and $0.649 \pm 0.026$ in the non-US irradiation group (Fig. 5a, b). The results indicated that the amount of biofilm was significantly reduced because of US irradiation $(p<0.01)$.

For the samples irradiated for $12 \mathrm{~h}$, the mean absorbance was $0.418 \pm 0.121$ in the US irradiation group and $0.567 \pm 0.160$ in the non-US irradiation group (Fig. 6a, b). There were no significant differences between the two groups. 
Fig. 3 Time course of ultrasound (US) irradiation on the biofilm in "Examination 2" (short-time irradiation). a Single irradiation, b Double irradiation

(a)

\section{Single-irradiation}

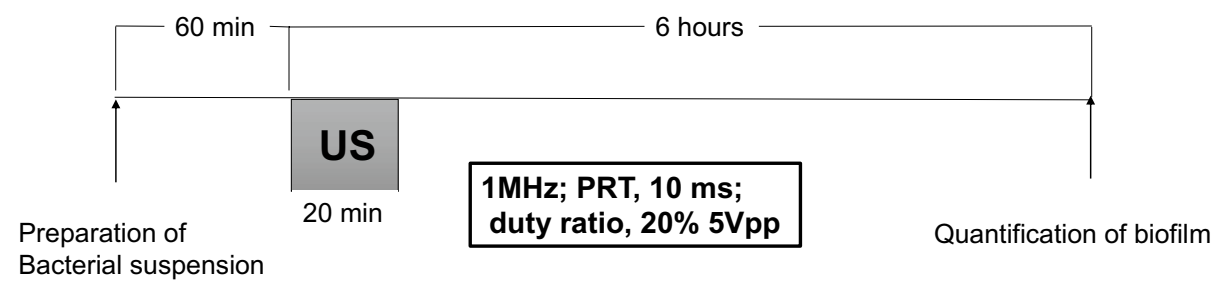

(b)

\section{Double-irradiation}

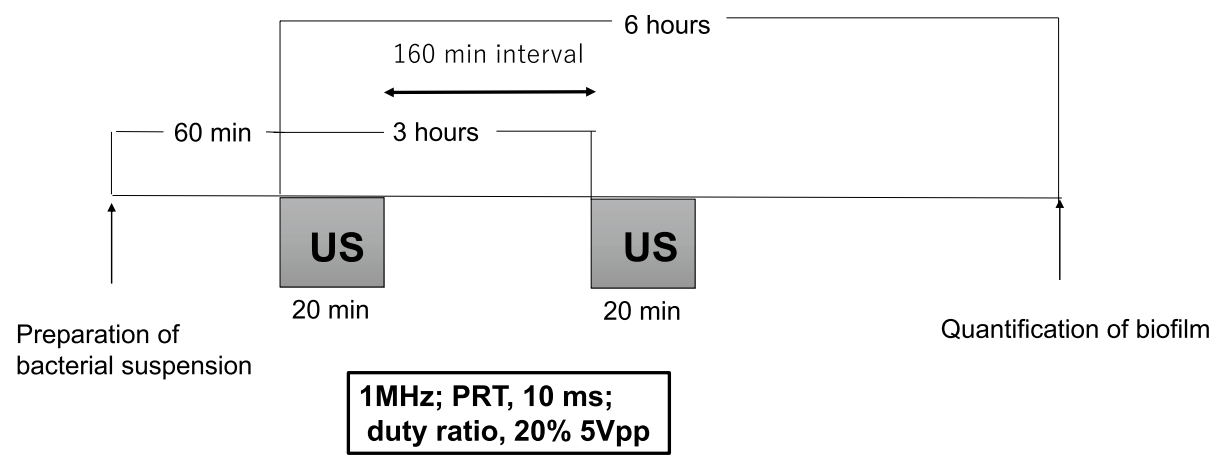

\section{Examination 2}

\section{Short-time single and double irradiation notably reduced biofilm formation}

In the case of single irradiation, the absorbance of samples was $0.218 \pm 0.018$ in the US irradiation group and $0.265 \pm 0.033$ in the non-US irradiation group. The reduction in biofilm formation was $17.9 \%$, and the difference was significant $(p<0.01)$. In the case of double irradiation, the absorbance of samples was $0.153 \pm 0.033$ in the US irradiation group and $0.230 \pm 0.045$ in the non-US irradiation group. The reduction in biofilm formation was $33.6 \%$. and the difference, in this case, was also significant $(p<0.01)$. Overall, in both groups, the amount of biofilm decreased notably because of US irradiation (Fig. 7a-c).

\section{Discussion}

The results of this study indicate that US irradiation has inhibitory effects on S. epidermidis biofilms.

Previous studies have shown US irradiation influences bacterial biofilms. The US intensity used in these studies was in the order of $\mathrm{W} / \mathrm{cm}^{2}$; however, this intensity is too high for clinical therapy applications. In this study, US intensities were $I_{\text {SATA }}=29 \mathrm{~mW} / \mathrm{cm}^{2}$ and $I_{\text {SATA }}=6 \mathrm{~mW} / \mathrm{cm}^{2}$ in Examination 1 and 2, respectively. This intensity is lower than the safe US exposure limit recommended by the FDA.

In addition, US irradiation was used for eradicating biofilm in previous studies, as well as in our study $[9,11]$. However, when a biofilm is disrupted, the bacteria present in and around the biofilm could disperse in the bloodstream, resulting in CRBS aggravation. In this study, it was demonstrated that adequate US irradiation could inhibit the formation of biofilm to some extent.

In "Examination 1", US irradiation for $24 \mathrm{~h}$ inhibited biofilm formation, but the same treatment for $12 \mathrm{~h}$ did not. This suggests that there is an optimal duration of US irradiation for inhibiting biofilm formation. An application time of $24 \mathrm{~h}$ would be inappropriate for hospitalized patients as the extended irradiation exposure may have adverse effects. Therefore, evaluating a shorter optimal duration for irradiation is important.

S. epidermidis biofilm formation occurs in two stages: rapid initial attachment of the bacteria to polymer surfaces and cell proliferation combined with the production of polysaccharide intercellular adhesion [5]. In "Examination 2", US irradiation $1 \mathrm{~h}$ after the preparation of bacterial 
(a) Sound pressure distribution in X direction
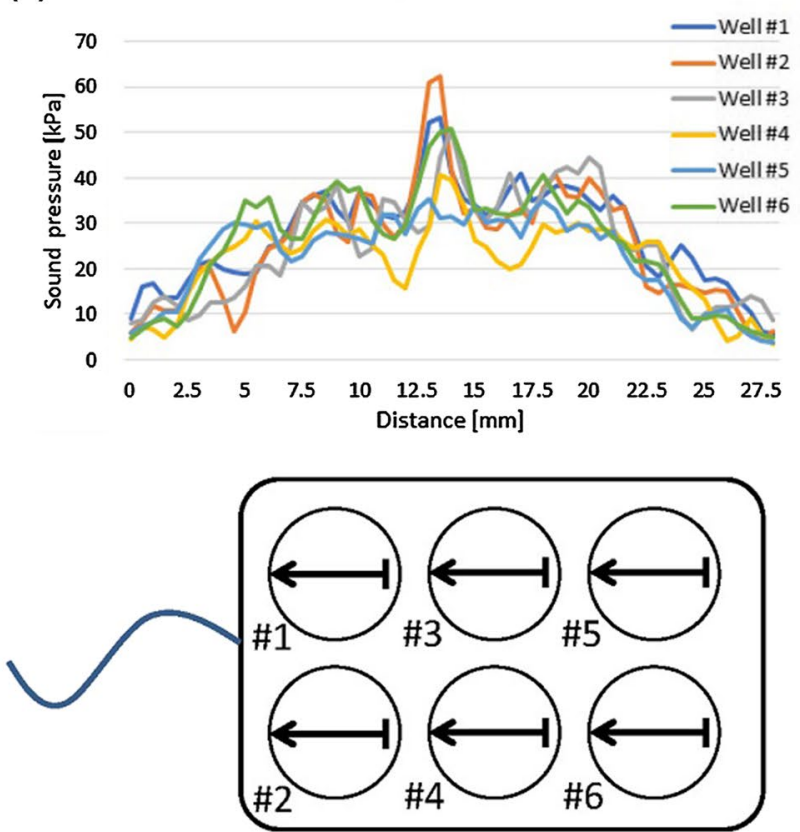

(b)

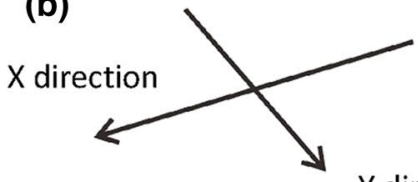

Y direction

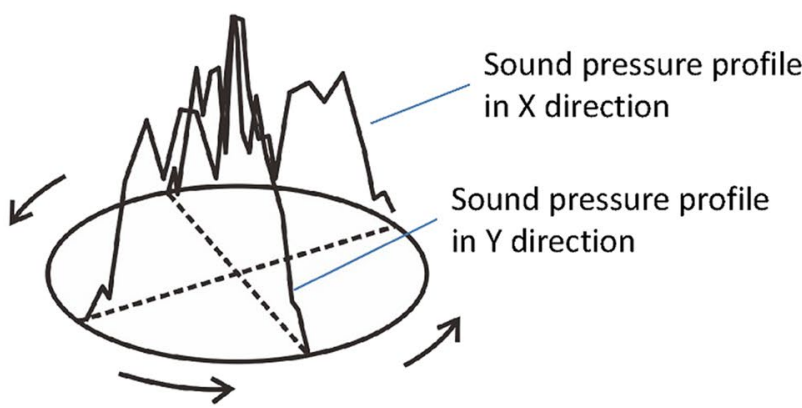

90 degree rotation

(d)

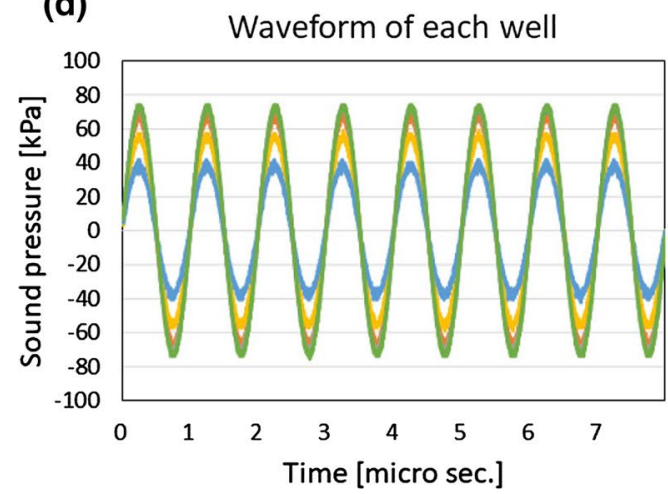

-Well \#1

-Well \#2

-Well \#3

-Well \#4

-Well \#5

-Well \#6
Sound pressure distribution in $Y$ direction
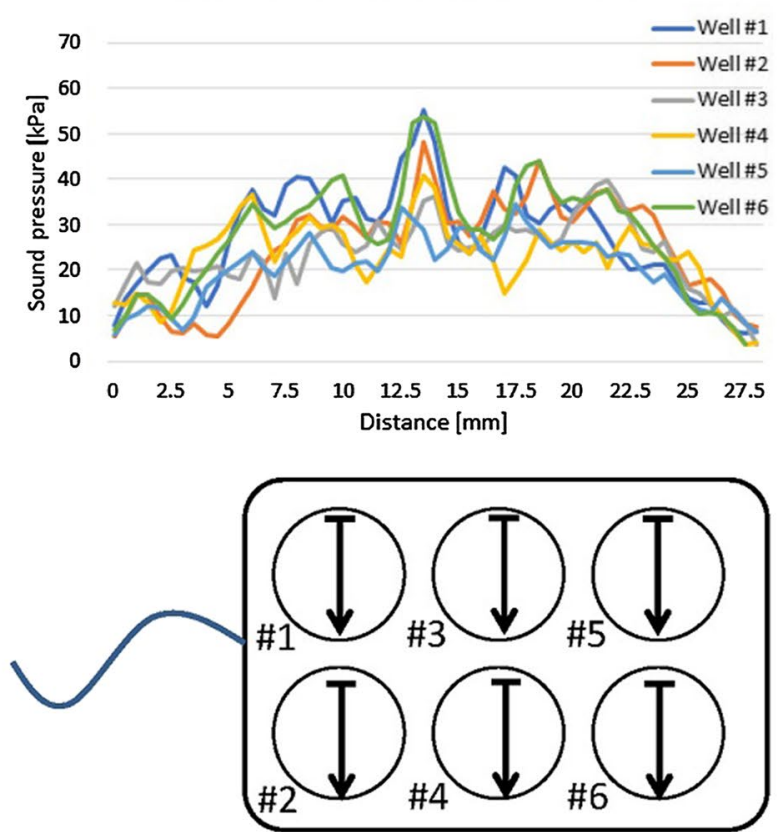

(c)

Sound intensity of each well

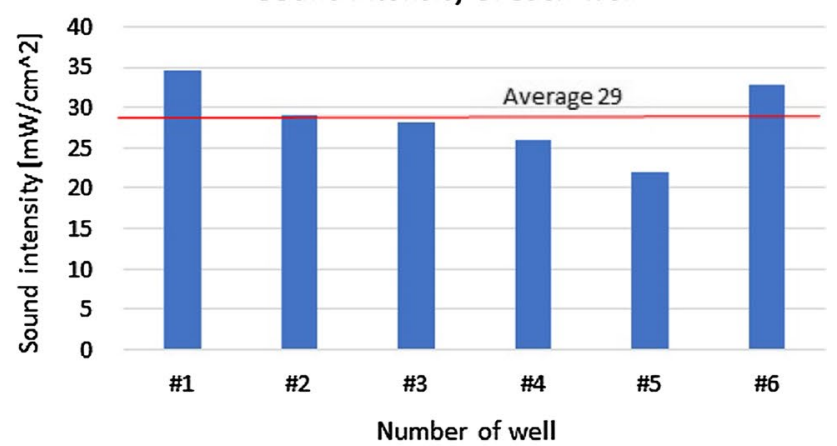

(e) Waveform at different positions in well \#2

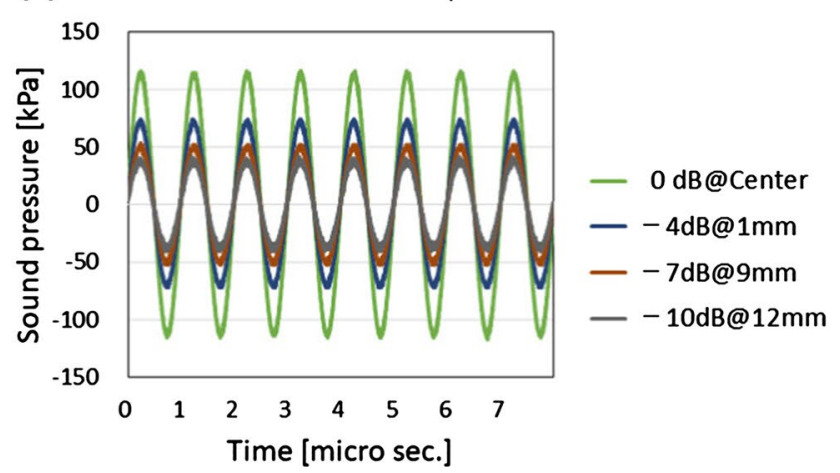


4Fig. 4 a The sound pressure distributions in the $X$ and $Y$ directions. b The total energy of US irradiation in each well was obtained by the rotation manner from the measured sound pressure profiles in both the $X$ and $Y$ directions as shown in Fig. 4b. $I_{\text {SATA }}$ was calculated as the total energy divided by the bottom area of the well. c Calculated sound intensity of each well. The average in the 6-well plate is 29 $\mathrm{mW} / \mathrm{cm}^{2}$. d The sound pressure waveform is observed at the center of each well. The waveforms are substantially sinusoidal. e The waveform at different positions (the center of the well and the peripheral positions) in the well. Every waveform even at different positions is also substantially sinusoidal
In the other studies $[14,15]$, a greater inhibitory effect was observed from US irradiation combined with antibiotics or microbubbles than that from US irradiation only. During US irradiation, microbubbles are formed, which may act on biofilms and increase their permeability to antimicrobial agents or even kill bacteria in biofilms [14, 15]. If US irradiation can be combined with antibiotics in clinical settings, US intensity can be reduced. In addition, the dose of antibiotics can be reduced, which is particularly beneficial
Fig. 5 a Images of biofilm on the bottoms of the 6-well plates in "Examination 1" (24-h irradiation). Left: US (+) plate. Right: US (-) plate. The amount of biofilm on the bottoms of US (+) plates was higher than that of US (-) plates. b Comparison of the amount of biofilm formed between the ultrasound (US) irradiation group and the nonUS irradiation group in "Examination 1" (24-h irradiation). The amount of biofilm formed was significantly lower in the US irradiation group (US +) than in the non-US irradiation group (US-) (a)

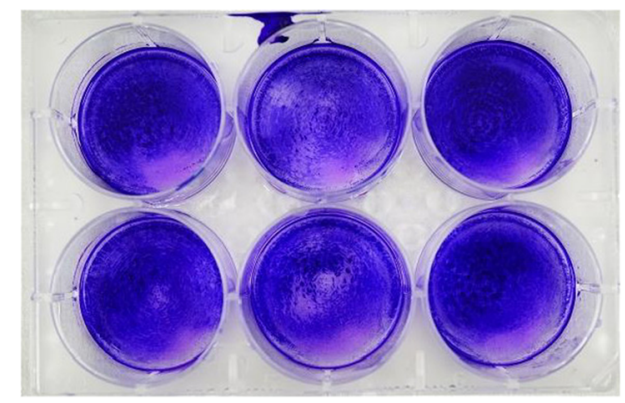

US (+)

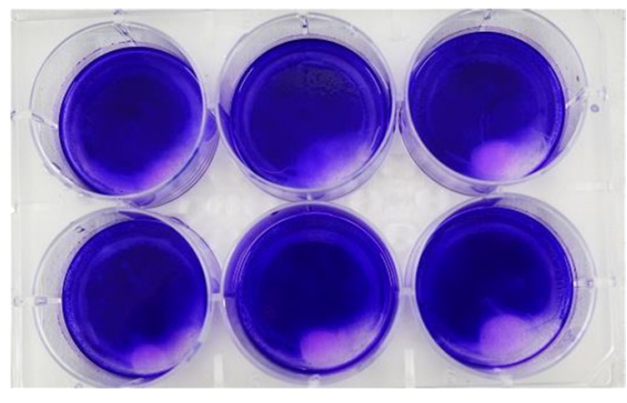

US (-)
Continuous wave for $24 \mathrm{hr}$

(b)

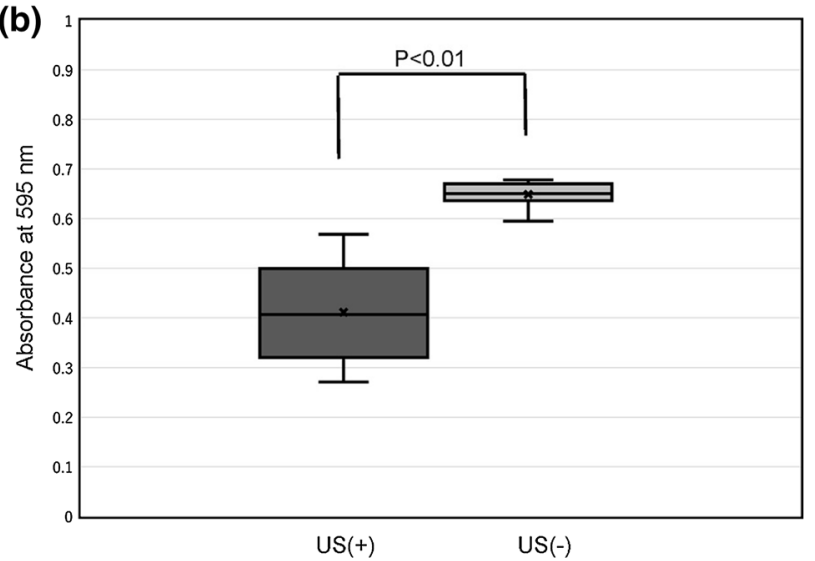

suspension had an inhibitory effect on biofilm formation. This result indicates that US irradiation has an effect on the early stage of biofilm formation, wherein bacteria attach to the bottom surface of the well. Our results concur with those of Wang $\mathrm{H}$ et al. [13], who stated that the key factor in stopping biofilm formation was to prevent bacteria from depositing on the medical device surface using ultrasonic vibration. In our study, US irradiation likely produced vibrations on the bottom surface of the well, and this vibration prevented the physical deposition of bacteria on the surface. for patients with chronic renal impairment or similar conditions who have a limitation on the dose of medications that can be administered.

There are two limitations to this study. First, the US frequency used in this study was only $1 \mathrm{MHz}$ because of the technical features of the SCI irradiator. If preventing bacteria from depositing on the surface by US vibration is key for the inhibition of biofilm formation, various US frequencies should be evaluated for their effect on biofilm formation. Second, the biofilm used in this study was grown in wells, 
Fig. 6 a Images of biofilm on the bottoms of the 6-well plates in "Examination 1" (12-h irradiation). Left: US (+) plate. Right: US(-) plate. There was no difference between the amount of biofilm on the bottoms of US (+) plates and US $(-)$ plates. b Comparison of the amount of biofilm formed between the ultrasound (US) irradiation group (US +) and the non-US irradiation group (US-) in "Examination 1" (12-h irradiation). The change in biofilm formation was not significant between the two groups (a)

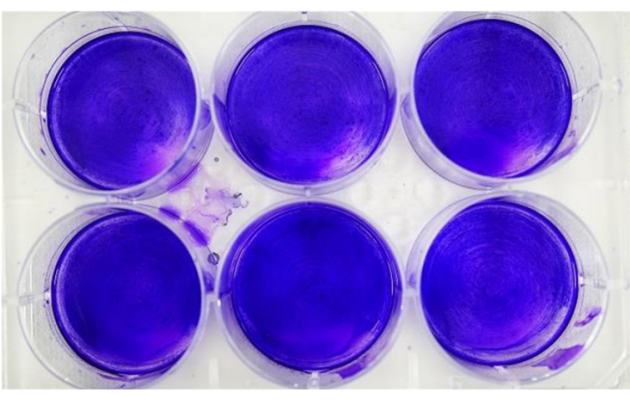

US(+)

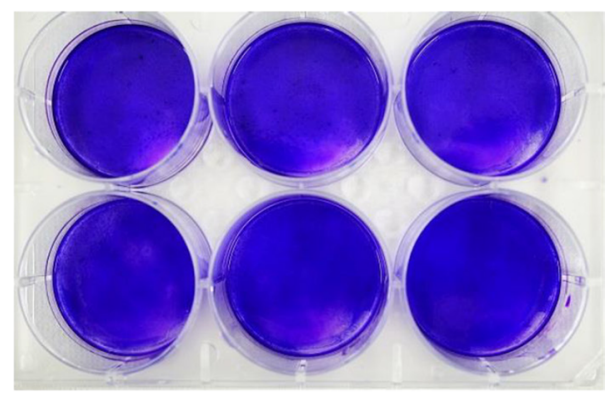

US(-)
Continuous wave for $12 \mathrm{~h}$

$\mathrm{P}=0.017$

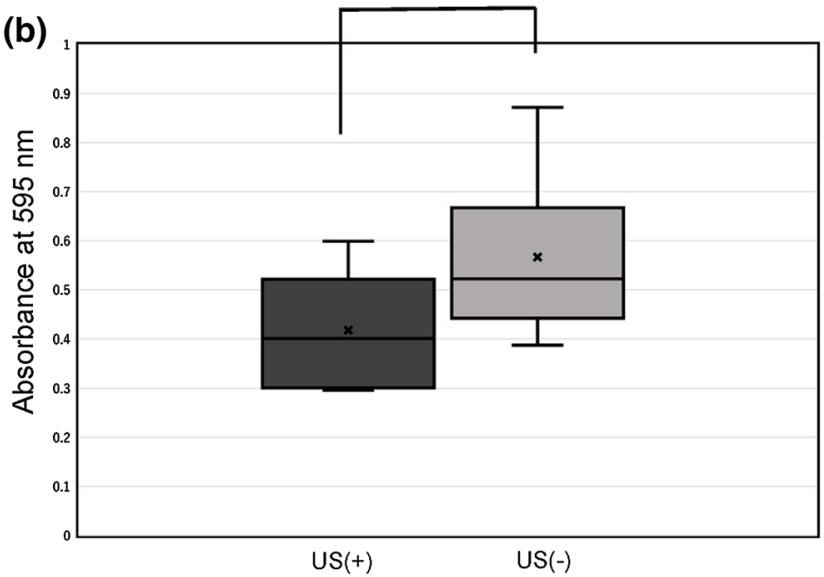

and not in a flow system. The goal of this study was to apply US irradiation to catheter-inserted vessels for the treatment of CRBSI. Hence, further studies must be conducted on the use of US irradiation on biofilms grown in the bloodstream. It is difficult to experimentally grow biofilm in the bloodstream; therefore, the development of in vivo systems for the formation of biofilm in the bloodstream may be necessary for adaption for therapy in a clinical setting.

In clinical settings, the necessary output power of the US generator will be expectedly higher than that in this study for the following two unavoidable reasons. First, the US is irradiated from the transducer to the catheter from the surface of the skin. In this case, the US wave on the catheter is the traveling wave, not the multiple echoes or the standing wave that were generated in this study. Second, the acoustically effective area on the catheter is smaller than that of the bottom of the well, because the cross-section of the catheter is round, while the bottom of the well is flat.

However, the effective sound intensity discovered in this study is sufficiently small to ensure the safety of living tissue. Hence, the possibility of clinical application can be fully expected. Future work will focus on investigating the most effective conditions for US irradiation and develop a practical method, such as irradiation of catheters, to solve clinical problems in patients suffering from CRBSI. 
(a)

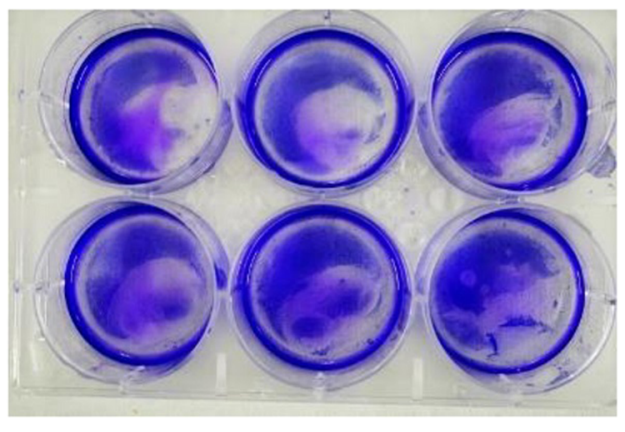

US (+)

Single irradiation (Pulse wave)

(b)

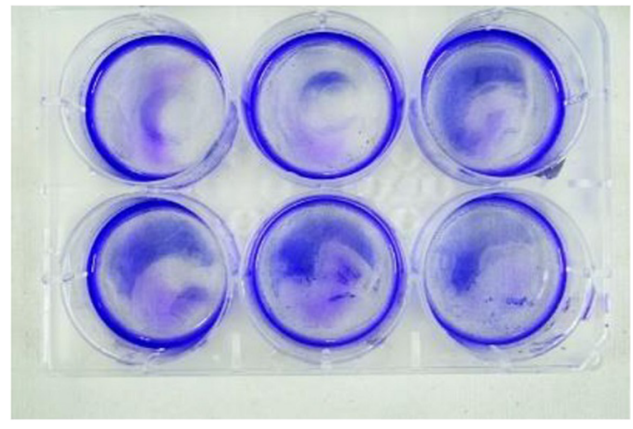

US $(+)$

Double irradiation (pulse wave)

(c)

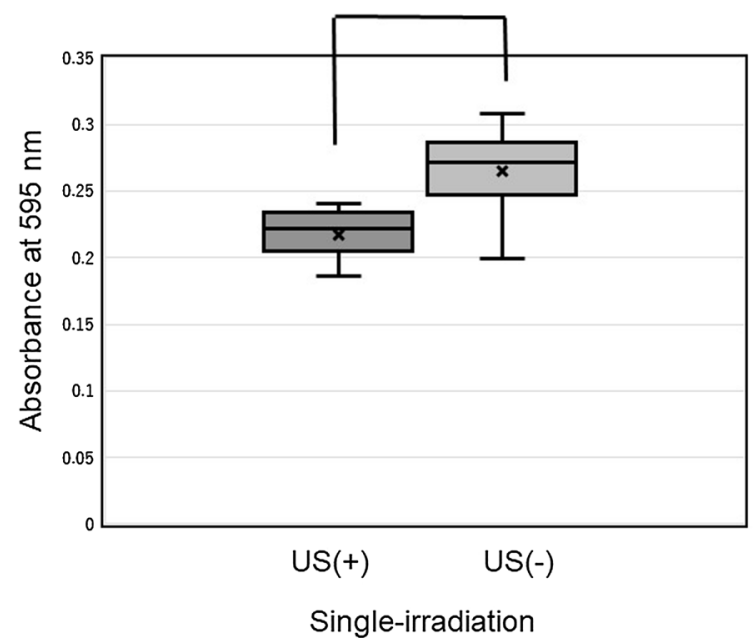

Fig. 7 a, b Images of biofilm on the bottoms of the 6-well plates in "Examination 2". a Single irradiation. b Double irradiation. Left: US $(+)$ plate. Right: US (-) plate. The amount of biofilm on the bottoms of US (+) plates was higher than that of US (-) plates. c Compari-

\section{Conclusion}

Continuous and pulsed wave US irradiation show potential for the inhibition of $S$. epidermidis biofilm formation at a level below the FDA's recommended therapeutic US

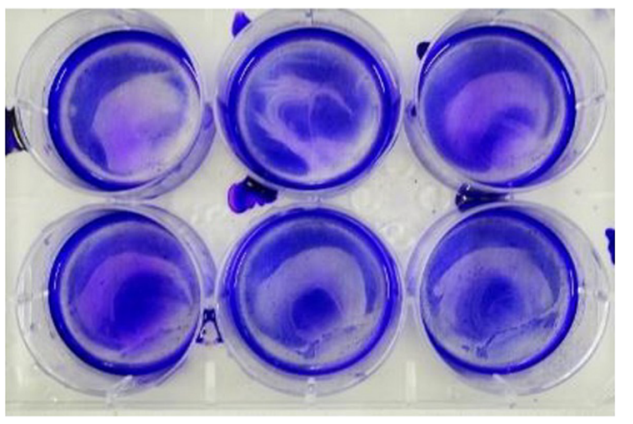

US(-)

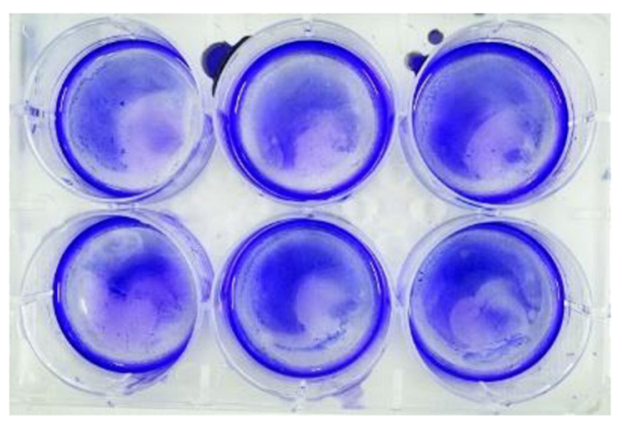

US(-)

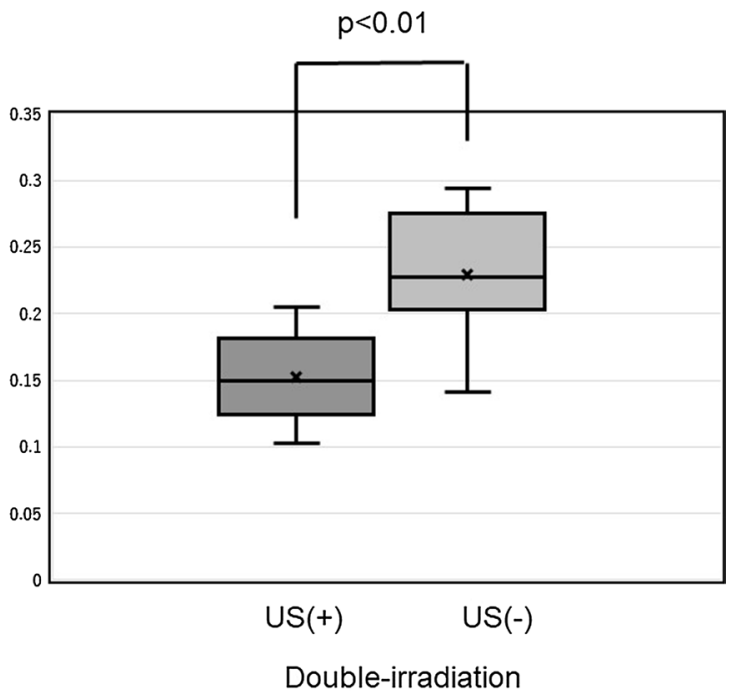

son of the amount of biofilm formed between the single and double ultrasound (US) irradiation groups (US +) and the non-US irradiation group (US-) in "Examination 2". The amount of biofilm formed was significantly lower in the single and double US irradiation groups

intensity level. Continuous-wave US irradiation significantly inhibited biofilm formation after $24 \mathrm{~h}$ of exposure, but not after $12 \mathrm{~h}$, while pulsed-wave US irradiation applied in two 20 -min intervals produced a significant $33.6 \%$ reduction in biofilm formation. US irradiation could be a novel method 
for preventing bacteria from forming biofilms, and this could be useful in treating CRBSIs caused by bacteria in biofilms.

Acknowledgements This research was supported by the Research and Development JSPS KAKENHI, Grant Number 18K18401. We would like to thank Editage (www.editage.com) for English language editing.

Author contribution $\mathrm{HK}$ and $\mathrm{YJ}$ : performing experiments and analyzing and interpreting data. YS and LC: deciding bacterial culture conditions and providing advice about the quantification of biofilms. TM and NT: deciding ultrasound irradiation parameters. TY: providing advice about the basic procedures of the laboratory work.

\section{Declarations}

Conflict of interest Harumi Koibuchi, Yasutomo Fujii, Yusuke Sato'o, Takashi Mochizuki, Toshiyuki Yamada, Longzhu Cui, and Nobuyuki Taniguchi declare that they have no conflicts of interest.

Ethics approval This article does not contain any studies with human or animal subjects performed by any of the authors.

Open Access This article is licensed under a Creative Commons Attribution 4.0 International License, which permits use, sharing, adaptation, distribution and reproduction in any medium or format, as long as you give appropriate credit to the original author(s) and the source, provide a link to the Creative Commons licence, and indicate if changes were made. The images or other third party material in this article are included in the article's Creative Commons licence, unless indicated otherwise in a credit line to the material. If material is not included in the article's Creative Commons licence and your intended use is not permitted by statutory regulation or exceeds the permitted use, you will need to obtain permission directly from the copyright holder. To view a copy of this licence, visit http://creativecommons.org/licenses/by/4.0/.

\section{References}

1. Lai NM, Lai NA, O'Riordan E, et al. Skin antisepsis for reducing central venous catheter-related infections. Cochrane Database Syst Rev. 2016;CD010140. https://doi.org/10.1002/14651858.CD010 140.pub2.
2. Frost SA, Hou YC, Lombardo L, et al. Evidence for the effectiveness of chlorhexidine bathing and health care-associated infections among adult intensive care patients: a trial sequential metaanalysis. BMC Infect Dis. 2018;18:679.

3. Gominet M, Compain F, Beloin C, et al. Central venous catheters and biofilms: where do we stand in 2017? APMIS. 2017; 125:365-75.

4. Kloos WE, Bannerman TL. Update on clinical significance of coagulase-negative staphylococci. Clin Microbiol Rev. 1994;1:117-40.

5. Mack D. Molecular mechanisms of Staphylococcus epidermidis biofilm formation. J Kosp Infect. 1999;43:S113-25.

6. Mah TF, O'Toole GA. Mechanism of biofilm resistance to antimicrobial agents. Trends Microbiol. 2001;9:34-9.

7. Buldakov MA, Hassan MA, Jawaid P, et al. Cellular effects of low intensity pulsed ultrasound and X-irradiation in combination in two human leukemia cell lines. Ultrason Sonochem. 2015;23:339-46.

8. Guidance for Industry and Food and Drug Administration Staff. Marketing Clearance of Diagnostic Ultrasound Systems and Transducers. https://www.fda.gov/media/71100/download.

9. Erriu M, Blus C, Szmukler-Moncler S, et al. Microbial biofilm modulation by ultrasound: current concepts and controversies. Ultrason Sonochem. 2014;21:15-22.

10. Vyas N, Manmi K, Wang Q, et al. Which parameters affect biofilm removal with acoustic cavitation? A Review. Ultrasound Med Biol. 2019;45:1044-55.

11. Koibuchi H, Fujii Y, Hirai Y, et al. Effect of ultrasonic irradiation on bacterial biofilms. J Med Ultrasonics. 2018;45:25-9.

12. Tabuchi Y, Sugahara Y, Ikegame M, et al. Genes responsive to low-intensity pulsed ultrasound in MC3T3-E1 preosteoblast cells. Int J Mol Sci. 2013;11:22721-40.

13. Wang H, Teng F, Yang X, et al. Preventing microbial biofilms on catheter tubes using ultrasonic guided waves. Sci Rep. 2017;7:1-9.

14. Wang J, Wen K, Liu X, et al. Multiple low frequency ultrasound enhances bactericidal activity of vancomycin against methicillinresistant Staphylococcus aureus biofilms. BioMed Res Int. 2018. https://doi.org/10.1155/2018/6023101 (eCollection 2018).

15. Dong Y, Li J, Li P, et al. Ultrasound microbubbles enhance the activity of vancomycin against Staphylococcus epidermidis biofilms in vivo. J Ultrasound Med. 2018;37:1379-87.

Publisher's Note Springer Nature remains neutral with regard to jurisdictional claims in published maps and institutional affiliations. 